\title{
O Problema da Minimização de Troca de Gramaturas e Rolos Corrugadores em uma Indústria de Embalagens de Papelão Ondulado
}

\author{
M. FERREIRA ${ }^{1 *}$ e S. A. DE ARAUJO ${ }^{2}$ \\ Recebido em January 18, 2019 / Aceito em February 15, 2021
}

\begin{abstract}
RESUMO. Neste trabalho abordamos um problema real identificado no processo produtivo de indústrias de embalagens de papelão ondulado. O setor de planejamento e controle da produção emite boletins que trazem as características relativas às chapas que serão produzidas. A produção destas chapas é feita no corrugador, onde bobinas são instaladas, de acordo com as características dos boletins. O problema surge da necessidade de minimizar trocas de gramaturas e rolos corrugadores durante o processo de produção das chapas com as características contidas nos boletins, uma vez que cada boletim traz as gramaturas específicas das camadas (geralmente são 3 ou 5) da chapa, além do tipo de onda empregada no(s) miolos(s). O sequenciamento dos boletins impacta na quantidade de trocas de gramaturas e rolos corrugadores. Interpretamos este problema como um Problema de Minimização de Trocas de Ferramentas e apresentamos quatro modelos matemáticos para o caso em estudo. Resultados computacionais são apresentados comparando os resultados obtidos pelos modelos matemáticos propostos e por uma fábrica do setor, indicando que é possível reduzir em até $37 \%$ o número de trocas de gramaturas.
\end{abstract}

Palavras-chave: programação inteira mista, minimização de trocas de ferramentas, indústria de embalagens.

\section{INTRODUÇÃO}

As embalagens de papelão ondulado são as mais utilizadas no mundo, por apresentarem baixo custo, serem recicláveis e otimizarem o uso do espaço. No Brasil, segundo dados do IBGE para o ano de 2019, as Indústrias de Embalagens de Papelão Ondulado (IEPO), movimentaram R \$ 13,3 bilhões, o que revela a força deste setor na economia. Uma embalagem de papelão é composta de um ou mais elementos ondulados (miolos) colados, através de um adesivo, a elementos planos (camadas). A ondulação de um miolo é feita por cilindros chamados rolos corrugadores.

\footnotetext{
*Autor correspondente: Marcelo Ferreira - E-mail: marcelo.ferreira@matematica.uftm.edu.br

${ }^{1}$ Universidade Federal do Triângulo Mineiro-UFTM, Av. Dr. Randolfo Borges Júnior, 1400, Univerdecidade, Uberaba, MG, Brasil - E-mail: marcelo.ferreira@matematica.uftm.edu.br https://orcid.org/0000-0002-6854-1837

${ }^{2}$ Universidade Estadual Paulista Júlio de Mesquita Filho-UNESP, Rua Cristóvão Colombo, 2265, Jardim Nazareth, São José do Rio Preto, SP, Brasil - E-mail: silvio.araujo@unesp.br https://orcid.org/0000-0002-4762-2048
} 
As gramaturas (peso do papel, expresso em gramas e que serve como termo de comparação entre os tipos de papel) das camadas e miolos são definidas de acordo com as características da embalagem a ser produzida.

O processo produtivo das IEPO tem uma cadeia complexa e vem chamando a atenção de inúmeros pesquisadores da área de otimização desde a década de 60, o que possibilitou a identificação e estudo de uma série de problemas interessantes. O mais comum deles é o problema da minimização da perda lateral também chamada de refile (Trim Loss Problem) que surge no processo de elaboração dos boletins pelo PCP (Planejamento e Controle de Produção). No boletim, busca-se as melhores combinações de embalagens contidas nos pedidos dos clientes, levando em conta a minimização de perdas laterais. O boletim traz informações acerca da quantidade de camadas, largura e gramatura das camadas e o(s) tipo(s) de onda(s) utilizada(s) no(s) miolo(s). Observe que as embalagens contidas num mesmo boletim devem ter as mesmas características em relação à quantidade e gramatura das camadas e miolo(s). Assim, o problema da minimização da perda lateral consiste em minimizar a diferença entre a largura da combinação das embalagens contidas no boletim e a largura das bobinas de papel utilizadas na produção. Este problema foi investigado em [12], [11], [15], [19] e [21], dentre outros. Em [22] os autores apresentam um modelo matemático para minimizar a perda lateral e inclui restrições de capacidade de armazenamento. Em [2] o autor estende o artigo de [22] considerando o trade-off entre perda lateral e preparação de máquina.

O problema da minimização das pilhas em aberto com foco no processo produtivo das IEPO foi investigado em [16], onde uma heurística foi introduzida de forma a minimizar o número de pedidos em aberto. A redução do número de pedidos em aberto é relevante devido às limitações no espaço de armazenamento de tais pedidos. O trabalho de [16] foi generalizado em [1], em que uma nova heurística é usada para calcular um limite superior das pilhas abertas, bem como um método exato é proposto. O problema da minimização do número de paragens do corrugador (também chamado de onduladeira, máquina multiestágio que mede $150 \mathrm{~m}$ e pesa 500 toneladas) é investigado em [17], onde são integrados também a minimização das perdas laterais e a minimização do comprimento das bobinas de papel utilizados.

Em [9], os autores consideram o problema de alocação de bobinas no corrugador para produzir as chapas de papelão ondulado demandadas, com o objetivo de minimizar o número de bobinas utilizadas, consumos parciais de bobinas, sobras não aproveitáveis de bobinas e paragens do corrugador. Em [4] é desenvolvido um software para uma versão simplificada deste problema.

Apesar da relevância no processo produtivo das IEPO, não encontramos trabalhos na literatura que tratem do Problema de Minimização de Trocas de Gramaturas e Rolos Corrugadores - PMTGRC. Este problema está relacionado ao sequenciamento dos boletins, visando a minimização do número de trocas de gramaturas e rolos corrugadores, que impactam nos custos de produção e na produtividade. Cada boletim tem gramaturas específicas para cada uma das camadas da chapa, devendo-se considerar ainda, o tipo de onda que será utilizada no miolo da chapa, sendo que cada tipo de onda é produzida por um rolo corrugador específico. 
Neste trabalho tentamos cobrir este gap com uma proposta de modelagem do PMTGRC como um Problema de Minimização de Trocas de Ferramentas (PMTF) ([23], [14] e [3]), também conhecido na literatura como SSP (do Inglês, Job Sequencing and Tool Switching Problem), ToSP (do inglês, Tool Switching Problem) e TSFM (do Inglês, Tool Switching on a Flexible Machine) ([20]). Esse problema consiste basicamente em determinar a melhor sequência de um conjunto de tarefas, para as quais temos que utilizar ferramentas específicas, de modo que sejam necessárias o menor número de trocas de ferramentas. A máquina que executa as tarefas tem um magazine (compartimento para conter as ferramentas) limitado e que geralmente não comporta todas as ferramentas necessárias para executar todas as tarefas, o que obriga as trocas das ferramentas. No PMTGRC encontrado nas IEPO temos que a produção de cada chapa relacionada a um boletim corresponderá a uma tarefa, cada bobina de determinada gramatura corresponderá a uma ferramenta e, o magazine corresponderá ao conjunto limitado de porta-bobinas presente no corrugator. A principal diferença entre o problema tratado neste trabalho e o PMTF é que a ordem de instalação das bobinas (ferramentas) é importante, pois, vão ser utilizadas para produzir determinada camada da chapa (tarefa).

Este trabalho tem como principais contribuições a apresentação do PMTGRC e a proposição de quatro modelos matemáticos diferentes para resolver este problema. Resultados computacionais são apresentados, com base em dados aleatórios e em dados reais, em que os modelos são comparados. Também é feita uma comparação entre um dos modelos e os resultados reais obtidos pela indústria estudada.

O artigo está organizado da seguinte forma: na Seção 2 é feita a descrição do processo produtivo em uma indústria de embalagens de papelão e a descrição do problema a ser investigado. Na Seção 3 são apresentados quatro modelos matemáticos e, na Seção 4, os resultados computacionais são apresentados. Na Seção 5 são incluídas as conclusões e trabalhos futuros.

\section{DEFINIÇÃO DO PROBLEMA}

O processo produtivo das IEPO pode ser dividido em três etapas. A primeira etapa ocorre na planta de papel, e consiste na produção das bobinas de papel de diferentes gramaturas que tem como matéria-prima as aparas (todos os tipos de papéis usados), e a pasta quimio-mecânica (pasta de materiais ligno-celulósicos prévia e levemente tratados com reagentes químicos, obtida por desfibramento a pressão atmosférica). As bobinas produzidas vão para o estoque de bobinas.

A segunda etapa ocorre no corrugador onde, de acordo com as especificações dos boletins, as bobinas produzidas na primeira etapa são inseridas aos pares, em estruturas denominadas portabobinas (Figura 1). Utilizando as bobinas nele instaladas, o corrugador produz grandes chapas retangulares de papelão ondulado. Posteriormente, estas chapas são cortadas em retângulos menores, chamados blanks, que constituem a base para a produção de uma caixa de papelão.

Finalmente, na terceira etapa, que ocorre na impressora lexicográfica, são feitos os recortes e impressão dos blanks. Alguns tipos de caixas, devido a sua especificação, podem passar por outras 


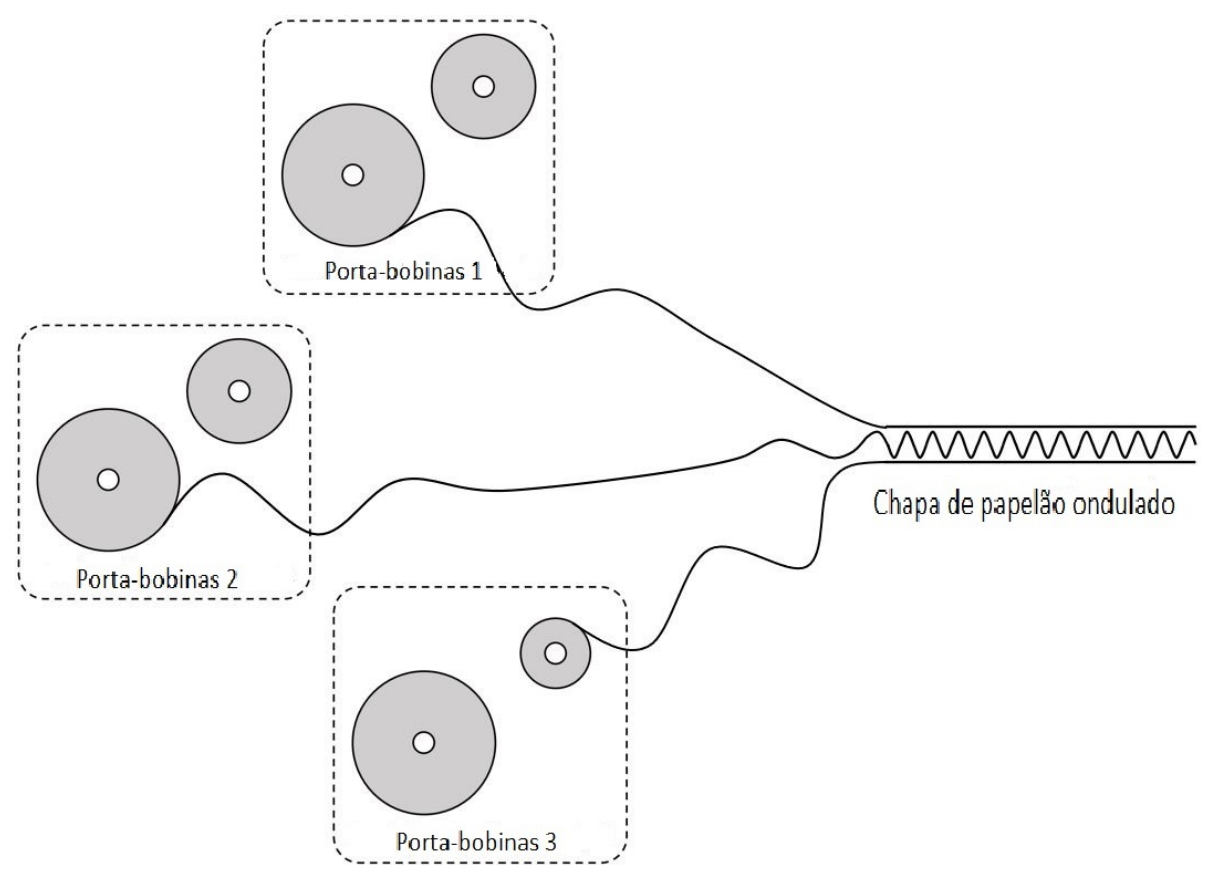

Figura 1: Ilustração de porta-bobinas para a produção de chapas com 3 camadas. Fonte [9].

etapas, como é o caso de caixas grampeadas ou com colagem especial. Etapas estas realizadas nas máquinas grampeadeiras e coladeiras.

O foco deste trabalho está na segunda etapa, mais especificamente, no sequencimento dos boletins a serem produzidos. Quando duas chapas, relacionadas a dois boletins, são produzidas sucessivamente e as gramaturas são diferentes para alguma de suas camadas, a sobra de bobina da primeira deverá ser retirada e transportada até o estoque, o que acarreta custos de transporte. Porém, quando as gramaturas são as mesmas para todas as camadas, pode-se enxergar as duas chapas como uma só o que facilita a programação do uso das bobinas e reduz os custos de transporte. Da mesma forma, se para duas chapas sucessivas os rolos corrugadores forem diferentes, deverá ser feita a reposição do rolo, que demanda tempo e implica em perda de produtividade. Obter a melhor sequência para os boletins de forma que sejam minimizadas as trocas de gramatura e rolos corrugadores é um problema relevante no processo produtivo das IEPO. Conforme observado anterioremente, não encontramos nenhum artigo na literatura que trate do problema de minimização de gramaturas e de rolos corrugadores.

Para maior clareza, sintetizamos na Tabela 1 alguns exemplos envolvendo boletins de chapas de parede simples ( 2 camadas e 1 miolo) e boletins de chapas parede dupla (3 camadas e 2 miolos). O vetor $(120,100,120)$ relativo ao boletim 1 , indica as gramaturas das duas camadas $(120$ e 120) e do miolo (100). Da mesma forma, o vetor $(160,120,160,120,160)$ relativo ao boletim 3 indica as gramaturas das três camadas $(160,160$ e 160) e dos miolos (120 e 120). Observe 
que, apesar do corrugador possuir 5 porta-bobinas, a chapa de papelão de parede simples será produzida utilizando somente os três primeiros porta-bobinas, relacionados às duas camadas e um miolo. $\mathrm{O}$ vetor $(\mathrm{B})$, relativo ao boletim 1 , indica o tipo de onda do miolo e o vetor $(\mathrm{B}, \mathrm{A})$, relativo ao boletim 3, indica o tipo de onda de cada miolo da chapa de parede dupla. Para o miolo, teremos duas informações associadas, a gramatura e o tipo de onda. Para simplificarmos o problema, consideraremos dois porta-bobinas adicionais fictícios, associados ao tipo de onda dos miolos. Assim, o boletim 2 por exemplo, seria denotado por (120,100,120,100,120,A,B). Estes vetores são ordenados, ou seja, as gramaturas e os tipos de ondas indicados devem ser utilizadas exatamente na ordem proposta.

Tabela 1: Exemplos de boletins com suas principais informações.

\begin{tabular}{|c|c|c|c|}
\hline Boletim & Tipo de papelão & Gramatura & Tipo de onda \\
\hline 1 & Parede simples & $(120,100,120)$ & $(\mathrm{B})$ \\
2 & Parede dupla & $(120,100,120,100,120)$ & $(\mathrm{A}, \mathrm{B})$ \\
3 & Parede dupla & $(160,120,160,120,160)$ & $(\mathrm{B}, \mathrm{A})$ \\
4 & Parede simples & $(140,120,140)$ & $(\mathrm{A})$ \\
5 & Parede dupla & $(140,100,140,100,140)$ & $(\mathrm{B}, \mathrm{B})$ \\
\hline
\end{tabular}

A forma que os boletins são sequenciados determinará o número de troca de gramaturas e o número de trocas de rolos corrugadores. Quando finaliza-se a produção da chapa relacionada a um boletim em um determinado porta-bobinas, pode-se ter um toco (sobra de bobina) em uma das camadas cuja gramatura é diferente daquela que deverá ser utilizada naquela camada da próxima chapa, relacionada ao próximo boletim. Neste caso, este toco deve ser devolvido ao estoque, o que implica em custo de transporte, ou deve ser descartado pois não poderá ser mais aproveitado, o que implica em perda de matéria-prima. Da mesma forma, se duas determinadas chapas relacionadas a dois boletins subsequentes tem, para um miolo, um tipo de onda diferente, o rolo corrugador (que imprime a onda no papel) deve ser substituído, e essa troca implica em perda de produtividade, pois o corrugador deve ser desligado durante a substituição.

Encontrar o melhor sequenciamento dos boletins de forma a minimizar as trocas de gramaturas e rolos corrugadores é um problema relevante no processo produtivo, pois tem impacto nos custos de produção e na produtividade. Por exemplo, considere a produção das chapas relacionadas aos boletins 2, 3 e 5, nesta ordem, teremos então 10 trocas de gramaturas e 3 trocas de rolos corrugadores, já para a sequência 2,5 e 3 , teremos 8 trocas de gramaturas e 2 trocas de rolo corrugador. Com o problema definido apresentaremos a seguir quatro propostas de modelagem matemática.

\section{MODELAGEM MATEMÁTICA}

Nesta seção propomos quatro modelos para o PMTGRC. Os modelos são inspirados na literatura referente ao PMTF, com adaptações necessárias para modelar o PMTGRC. Tais adaptações são originais e surgem, principalmente, devido ao fato de que a ordem de instalação das bobi- 
nas (ferramentas) nos porta-bobinas (magazine) é importante, pois cada bobina é instalada num porta-bobinas específico e vai ser utilizada para produzir determinada camada da chapa (tarefa). É importante observar que quadro modelos diferentes são propostos com o objetivo de comparar o desempenho destes modelos na obtenção de soluções para o PMTGRC.

\subsection{Modelo 1}

O modelo de programação inteira proposto a seguir foi baseado no proposto por [23].

Considere os seguintes conjuntos e variáveis:

\section{Conjuntos:}

- $T$ : Conjunto dos boletins $\{1, \ldots, n\}$ (índices $j, k$ ). Os índices $k$ e $j$ serão usados em diferentes contextos para indicar a posição do boletim no sequenciamento e $n$ representa o número total de boletins.

- $F$ : Conjunto com tipos de gramaturas e tipos de rolos corrugadores $\{1, \ldots,|F|\}$ (índice $t$ ).

- $P$ : Conjunto dos porta-bobinas $\{1, \ldots,|P|\}$ (índice $p$ ). Conforme observado anteriormente, consideramos $|P|=7$ (5 gramaturas e 2 rolos corrugadores).

- $T_{t p}$ : Conjunto de boletins que utilizam gramatura ou o rolo corrugador $t$ no porta-bobinas $p\left\{1, \ldots,\left|T_{t p}\right|\right\}$ (índice $j$ ).

\section{Parâmetro:}

- $c$ : Proporção em que a troca de rolos currugadores é mais cara do que a troca de gramaturas.

\section{Variáveis:}

- $w_{j k}$ : Variável binária.Vale 1 se a chapa associada ao boletim $j$ é processada na $k$-ésima posição do sequenciamento e vale 0 caso contrário.

- $y_{p k t}$ : Variável binária. Vale 1 se a gramatura ou o rolo corrugador $t$ está alocado no portabobina $p$ durante o processamento da chapa do $k$-ésimo boletim e vale 0 caso contrário.

- $z_{p k t}$ : Variável binária. Vale 1 se a gramatura ou o rolo corrugador $t$ está alocado no porta-bobina $p$ durante o processamento da chapa do $k$-ésimo boletim, mas não no processamento da chapa do $(k-1)$-ésimo boletim e vale 0 caso contrário.

O modelo proposto é o seguinte (Modelo 1):

$$
\min \sum_{p=1}^{5} \sum_{k=2}^{n} \sum_{t \in F} z_{p k t}+\sum_{p=6}^{7} \sum_{k=2}^{n} \sum_{t \in F} c \cdot z_{p k t}
$$




\section{Sujeito a:}

$$
\begin{gathered}
\sum_{j \in T} w_{j k}=1 ; k=1, \ldots, n . \\
\sum_{k=1}^{n} w_{j k}=1 ; j \in T . \\
\sum_{j \in T_{t p}} w_{j k} \leq y_{p k t} ; t \in F, p \in P, k=1, \ldots, n . \\
\sum_{t \in F} y_{p k t}=1 ; p \in P, k=1, \ldots, n . \\
y_{p k t}-y_{p k t-1} \leq z_{p k t} ; t \in F, p \in P, k=2, \ldots, n . \\
w_{j k}, y_{p k t}, \quad z_{p k t} \in\{0,1\}, j \in T, t \in F, p \in P, k=1, \ldots, n .
\end{gathered}
$$

A função objetivo (3.1) visa minimizar o número de trocas de gramaturas (primeira parcela) e rolos corrugadores (segunda parcela), sendo que segundo dados reais, a relação entre custo de trocas de gramaturas e rolos corrugadores é $1: c$. As restrições (3.2) e as restrições (3.3) dizem respeito à designação dos boletins, impondo que as chapas de todos os boletins devem ser processadas, uma por vez. As restrições (3.4) garantem que as gramaturas ou rolos corrugadores relacionadas às chapas de cada boletim estão nos devidos porta-bobinas antes do processamento da chapa. As restrições (3.5) garantem que para cada um dos porta-bobinas temos um único tipo de gramatura ou rolo corrugador. As restrições (3.6) computam as trocas de gramaturas e rolos corrugadores para a produção de chapas de dois boletins consecutivos. Em (3.7) temos os domínios das variáveis.

Com base em [20], apresentamos a seguir uma proposição que mostra a qualidade ruim da relaxação linear deste modelo, o que nos motivou a explorar outros modelos com relaxações lineares mais fortes. Observe que, a qualidade da relaxação linear é importante para a resolução dos modelos utilizando métodos embutidos em pacotes computacionais, como será feito neste artigo.

Proposição 3.1. Se $w_{j k}=\frac{1}{n}, \forall j \in T$ e $k=1, \ldots, n$, então a relaxação linear do Modelo 1 admite solução que não requer trocas de gramaturas ou rolos corrugadores.

Demonstração. Ao supormos que $w_{j k}=\frac{1}{n}, \forall j \in T$ e $k=1, \ldots, n$, satisfazemos as restrições (3.2) e (3.3) de designação, e a restrição (3.4) nos leva a:

$$
\sum_{j \in T_{t p}} \frac{1}{n} \leq y_{p k t} ; \forall t \in F, p \in P, k=1, \ldots, n \Rightarrow \frac{\left|T_{t p}\right|}{n} \leq y_{p k t} ; \forall t \in F, p \in P, k=1, \ldots, n .
$$


Seja $y_{p k t}=\frac{\left|T_{t p}\right|}{n}, \forall j \in T, p \in P, k=1, \ldots, n$. Isso é possível pois o restante das restrições são respeitadas. As restrições (3.5) são satisfeitas, pois $\sum_{t \in F} \frac{\left|T_{t p}\right|}{n}=\frac{\left|T_{1 p}\right|}{n}+\frac{\left|T_{2 p}\right|}{n}+\cdots+\frac{\left|T_{n p}\right|}{n}=\frac{n}{n}=1$, $\forall p \in P$ e $\forall k=1, \ldots, n$. Sendo assim, a restrição (3.6) unida ao objetivo de minimização nos leva a $z_{k t}=0, \forall j \in T$ e $k=1, \ldots, n$.

\subsection{Modelo 2}

No intuito de obter resultados computacionais melhores, propomos um outro modelo, baseado em [14], e que como o tal utiliza a estrutura do problema do caixeiro viajante, na qual após inserir um boletim fictício $\{0\}$, que represente o começo e o fim das operações, a sequência de processamento dos boletins representa um tour. A semântica das variáveis nessa formulação trata da consecutividade de pares de boletins. O modelo a seguir será denotado por Modelo 2.

Considere os seguintes conjuntos e variáveis:

\section{Conjuntos:}

- $T$ : Conjunto dos boletins $\{1, \ldots, n\}$ (índices $r, k$ ). Observe que $k$ e $r$ indicam a posição do boletim no tour e $n$ representa o número total de boletins.

- $F_{p k}$ : Conjunto de gramaturas ou rolo corrugador requerido pelo boletim $k$ no porta-bobinas $p\left\{1, \ldots,\left|F_{p k}\right|\right\}$ (índice $t$ ).

\section{Variáveis:}

- $x_{k r}$ : Variável binária.Vale 1 se a chapa associada ao boletim $k$ é seguido pela chapa associada ao boletim $r$.

- $z_{p k t}$ : Variável binária. Vale 1 se a gramatura ou o rolo corrugador $t$ é inserido no portabobina $p$ durante o processamento da chapa do $k$-ésimo boletim e vale 0 caso contrário.

O modelo proposto é o seguinte (Modelo 2):

$$
\min \sum_{p=1}^{5} \sum_{k=2}^{n} \sum_{t \in F_{p k}} z_{p k t}+\sum_{p=6}^{7} \sum_{k=2}^{n} \sum_{t \in F_{p k}} c \cdot z_{p k t}
$$

\section{Sujeito a:}

$$
\begin{gathered}
\sum_{r=0, k \neq r}^{n} x_{k r}=1 ; k=0, \ldots, n . \\
\sum_{k=0, k \neq r}^{n} x_{k r}=1 ; r=0, \ldots, n .
\end{gathered}
$$




$$
\begin{gathered}
y_{p k t}=1 ; p \in P, k=1, \ldots, n, t \in F_{p k} . \\
y_{p k t}=0 ; p \in P, k=1, \ldots, n, t \in F \backslash F_{p k} . \\
x_{k r}+y_{p r t}-y_{p k t} \leq z_{p r t}+1 ; p \in P, k=0, \ldots, n, r=1, \ldots, n, t \in F . \\
\sum_{k, r \in S} x_{k r} \leq|S|-1 ; k, r \in S \subset\{0,1, . . n\} . \\
\sum_{t \in F \backslash F_{p k}} z_{p k t}=0 ; p \in P, k=1, \ldots, n . \\
x_{k r}, y_{p k t}, z_{p k t} \in\{0,1\}, p \in P, k=1, \ldots, n, r=1, \ldots, n, t \in F .
\end{gathered}
$$

A função objetivo (3.8) visa minimizar o número de trocas de gramaturas (primeira parcela) e rolos corrugadores (segunda parcela). As restrições (3.9) e (3.10) dizem respeito à designação dos boletins, impondo que as chapas relacionadas a todos os boletins sejam processadas, uma por vez. As restrições (3.11) garantem que toda gramatura requerida por um boletim em um determinado porta-bobinas será alocada. As restrições (3.12) garantem que nenhuma outra gramatura ou rolo corrugador diferente da estabelecida, pode ser alocada em um determinado porta-bobinas. As restrições (3.13) se referem a contagem das trocas de gramaturas ou rolos corrugadores, pois se uma determinada gramatura ou rolo corrugador $t$ não está no porta-bobinas $p$ para a produção da chapa relativa ao boletim $k$, e nem foi inserida para a produção da chapa relativa ao boletim $r$, então esta não pode seguir aquela e requerer a gramatura ou rolo corrugador $t$ ao mesmo tempo. As restrições (3.14), DFJ ([6]), garantem que não há formação de subciclos. As restrições (3.15) tratam da eliminação de simetrias, proibindo que uma determinada gramatura ou rolo corrugador seja alocada sem ser necessária para produção da chapa do próximo boletim. Em (3.16) temos o domínio das variáveis.

\subsection{Modelo 3}

Se substituirmos as restrições (3.14) do Modelo 2, pelas restrições (3.17) e (3.18) de eliminação de sub-rotas propostas por [18], adaptadas para o caso em que temos um vértice (boletim) fictício, obtemos o modelo, denotado por (Modelo 3):

\section{Variáveis Adicionais:}

- $u_{k}$ : Variável inteira $\geq 1 \mathrm{e} \leq n+1$. Variável auxiliar utilizada na eliminação de sub-rotas. 


$$
\begin{gathered}
u_{k}-u_{r}+(n+1) \cdot x_{k r} \leq n ; k, r=1, \ldots, n, k \neq r . \\
1 \leq u_{k} \leq n+1 ; k=1, \ldots, n .
\end{gathered}
$$

Enquanto o número de restrições em (3.14) é exponencial, o número de restrições em (3.17) e (3.18) é polinomial.

\subsection{Modelo 4}

Neste modelo utilizamos a estrutura do problema do caixeiro viajante associada a um préprocessamento, em que calculamos as trocas de gramaturas e rolos corrugadores necessárias para a produção das chapas, de quaisquer dois boletins consecutivos. Dados dois boletins quaisquer de um conjunto com $n$ boletins, determinamos antecipadamente (antes de resolver o modelo), para cada porta-bobinas, se há troca de gramatura e rolo corrugador na produção de cada camada das chapas associadas ao par de boletins (veja o parâmetro $c o_{p k r}$ definido mais à frente). Observe que, dados dois boletins, a ordem em que suas chapas são produzidas não altera o número de trocas de gramaturas ou rolos corrugadores, o número de pares de boletins a serem analisados no pré-processamento para um conjunto com $n$ boletins se reduz a um cálculo combinatório simples, a saber, $C_{n, 2}=\frac{n \cdot(n-1)}{2}$. A função $c o_{p k r}$ associa 1 quando há troca de gramatura ou rolo corrugador para um determinado porta-bobinas entre duas chapas relativas a dois boletins e 0 caso contrário.

Este pré-processamento nos permite eliminar as variáveis $y_{p k t}$ e $z_{p k t}$ e as restrições (3.11), (3.12), (3.13) e (3.15) (Modelos 2 e 3), pois tais variáveis e restrições estão relacionadas ao computo das trocas, o que não é mais necessário no Modelo 4.

Considere os seguinte parâmetro e variável:

\section{Parâmetro:}

- $c o_{p k r}$ : Indica se há troca de gramatura ou rolo corrugador em determinada camada entre duas chapas relativas a dois boletins consecutivos $k$ e $r$ no porta-bobinas $p$.

\section{Variável:}

- $u_{k}$ : Variável inteira $\geq 0 \mathrm{e} \leq n$. Variável auxiliar utilizada na eliminação de sub-rotas, onde $u_{k}$ determina a ordem em que o vértice $k$ é visitado no tour.

O modelo proposto é o seguinte (Modelo 4):

$$
\min \sum_{p=1}^{5} \sum_{k=1}^{n} \sum_{r=1}^{k} c o_{p k r} \cdot x_{k r}+\sum_{p=6}^{7} \sum_{k=1}^{n} \sum_{r=1}^{k} c \cdot c o_{p k r} \cdot x_{k r}
$$


Sujeito a: (3.9) e (3.10)

$$
\begin{gathered}
u_{k}-u_{r}+(n-1) \cdot x_{k r}+(n-3) \cdot x_{r k} \leq n-2 ; k=2, \ldots, n, r=2, \ldots, n . \\
1+(n-3) \cdot x_{k 1}+\sum_{r=2}^{n} x_{r k} \leq u_{k} ; k=2, \ldots, n . \\
n-1-(n-3) \cdot x_{1 k}-\sum_{r=2}^{n} x_{k r} \geq u_{k} ; k=2, \ldots, n . \\
x_{k r}, \quad u_{k} \in Z, k=1, \ldots, n, \quad r=1, \ldots, n .
\end{gathered}
$$

A função objetivo (3.19) visa minimizar o número de trocas de gramaturas (primeira parcela) e rolos corrugadores (segunda parcela). As restrições (3.20), (3.21) e (3.22) são as restrições de eliminação de sub-rotas de ordem polinomial propostas por [7] e que são uma melhoria das restrições propostas por [18]. As restrições (3.20) fortalecem as restrições MTZ utilizando a técnica do lifting e as restrições (3.21) e (3.22) são inequações válidas para o MTZ, que juntas fortalecem a relaxação linear. Em (3.23) temos o domínio das variáveis.

A seguir apresentamos a Tabela 2 com o número de variáveis e restrições de cada um dos modelos. Além de apresentar estes números de maneira genérica, consideraremos dois exemplos para facilitar a comparação dos modelos:

- Exemplo $A: n=8,|F|=10,|P|=7$

- Exemplo $B: n=30,|F|=10,|P|=7$

Tabela 2: Número e variáveis e restrições dos modelos.

\begin{tabular}{|c|c|c|c||c|c|c|}
\hline Modelos & Variáveis Inteiras & Exemplo A & Exemplo B & Restrições & Exemplo A & Exemplo B \\
\hline Modelo 1 & $n[2+|P|(1+2|F|)]+|F||P|$ & 1184 & 5100 & $n(n+2|P||F|)$ & 1262 & 4540 \\
Modelo 2 & $n[2+|P|(2+|F-1|)]+2^{n}$ & 1184 & 5100 & $n(n+2|P||F|)$ & 903 & $1 \times 10^{9}$ \\
Modelo 3 & $n[2+|P|(2+|F-1|)]+2+n^{2}$ & 1192 & 5130 & $n(n+2|P||F|+1)$ & 698 & 3272 \\
Modelo 4 & $n(n+2)+1$ & 72 & 930 & $n(n+1)$ & 81 & 961 \\
\hline
\end{tabular}

Apresentaremos na próxima seção alguns resultados computacionais com o intuito de compreender a eficiência e as limitações de cada modelo proposto.

\section{RESULTADOS COMPUTACIONAIS}

Nesta seção apresentamos uma série de testes computacionais com instâncias geradas com base em dados reais. Inicialmente, descrevemos os principais parâmetros que caracterizam as instâncias. Na sequência, comparamos as soluções obtidas pelos quatro modelos propostos para 
um conjunto de instâncias aleatórias. Além disso, comparamos as soluções obtidas via modelo com soluções reais e processadas na prática para um conjunto de instâncias obtidas em uma IEPO visitada.

Os modelos matemáticos, todos de programação inteira, foram escritos na sintaxe da linguagem de modelagem AMPL (A Mathematical Programming Language)([10]) e resolvidos com o método branch-and-cut contido no resolvedor CPLEX 12.7 ([13]). O tempo máximo de processamento foi fixado em 600 segundos para cada instância. Os testes computacionais foram realizados utilizando um computador com processador Intel Core i7-2600, CPU de $3.4 \mathrm{GHz}$ e 16 GB memória RAM.

\subsection{Geração e caracterização dos dados}

Como citado anteriormente, o PMTGRC é similar ao PMTF, para o qual já existia um conjunto de instâncias propostas inicialmente por [5] e posteriormente por [24]. Devido às características próprias do PMTGRC não foi possível a utilização direta destas instâncias, porém a essência do processo de geração, descrita em [20] foi aproveitada.

Para o PMTF existem três parâmetros fundamentais: tarefas, ferramentas e capacidade do magazine e, devido a semelhança com o PMTGRC, foi possível estabelecer um paralelo utilizando os seguintes parâmetros: boletins, gramaturas/rolos corrugadores e quantidade de porta-bobinas.

Estabelecemos três grupos de testes denotados por 1, 2 e 3, que indicam respectivamente, a programação de um, dois ou três turnos de 8 horas de trabalho e como em cada turno processa-se em média, de 8 a 10 chapas, a quantidade mínima e máxima de boletins em cada grupo também ficou definida. Fixamos em 10 o número de instâncias geradas em cada grupo, em seguida, escolhemos o tipo de papelão a ser produzido, indicando a opção de instâncias com apenas chapas de papelão de parede simples por (S) e apenas chapas de papelão de parede dupla por (D). Finalmente, geramos aleatoriamente os tipos de gramaturas e os rolos corrugadores para cada instância, de acordo com os padrões estabelecidos na Tabela 3, que traz um resumo dessas informações. As instâncias geradas estão disponibilizadas no site: https://github.com/araujosaa/corrugated.

Tabela 3: Características das instâncias do PMTGRC.

\begin{tabular}{|c|c|c|c|}
\hline Grupos & Quantidade de boletins & Tipos de papelão & Gramaturas ou Rolos Corrugadores \\
\hline 1 & 08 a 10 & S, D & $100,120,140,160,180,200, \mathrm{~A}, \mathrm{~B}, \mathrm{C}, \mathrm{E}$ \\
2 & 16 a 20 & S, D & $100,120,140,160,180,200, \mathrm{~A}, \mathrm{~B}, \mathrm{C}, \mathrm{E}$ \\
3 & 24 a 30 & S, D & $100,120,140,160,180,200, \mathrm{~A}, \mathrm{~B}, \mathrm{C}, \mathrm{E}$ \\
\hline
\end{tabular}

Fonte: [8]. 
Consideramos então os seguintes parâmetros fundamentais:

a) Grupos - definimos três tipos de grupos: 1, que indica a programação de um turno de trabalho; 2, que indica a programação de dois turnos de trabalho e 3 , que indica a programação de três turnos de trabalho.

b) Tipos de papelão - definimos dois tipos: S, indica que a instância é formada por boletins cujas chapas tem parede simples e D que indica que a instância é formada por boletins cujas chapas tem parede dupla.

Teremos então 6 classes para análise, que serão denotadas pelo grupo e pelo tipo de papelão, nesta ordem, por exemplo: 1S indica o grupo 1 com boletins de parede simples (S). Como em cada classe temos 10 instâncias, teremos um total de 60 instâncias para serem analisadas na Seção 4.2. Além disso, temos a Seção 4.3 em que são apresentados resultados baseados em dados reais.

Por fim, para estabelecer o parâmetro $c$, relativo à proporção em que a troca de rolos currugadores é mais cara do que a troca de gramaturas, utilizamos o seguinte procedimento: supondo que uma troca de gramatura implique que a sobra da bobina seja devolvida ao estoque, o custo associado a essa troca foi calculado junto ao pessoal da empresa e vale $\mathrm{R} \$ 9,46$ e, para a troca de rolo corrugador, em que deve-se parar o corrugador o que consome em média 5 minutos, gera-se uma perda de produtividade de $\mathrm{R} \$ 2402,10$. Baseado nesses valores estabelecemos a relação de pesos entre troca de gramaturas e trocas de rolos corrugadores, na razão de 1:250.

\subsection{Comparando os modelos com dados gerados aleatoriamente}

Nessa seção compararemos na Tabela 4 os resultados obtidos para os Modelos 1, 2, 3 e 4 para as 6 classes descritas na Tabela 3. Analisaremos os valores mínimos (Min), máximos (Max) para as trocas de gramaturas e rolos corrugadores e valores mínimos (Min), máximos (Max) e médios $(\bar{M})$ para F.O. (ou seja, para os valores das soluções inteiras factíveis obtidas pelos modelos), tempo de processamento (T) e GAP para as 10 instâncias de cada classe. Este GAP é fornecido pelo resolvedor CPLEX e é calculado por $G A P=\left((L S-L I) /\left(e^{-10}+|L S|\right)\right.$, em que, $L S$ representa o menor limitante superior (solução inteira factível) e $L I$ o maior limitante inferior, ambos os limitantes obtidos pelo resolvedor CPLEX. Quando, para uma determinada instância, o valor do GAP é menor que $0,01 \%$ dizemos que o resolvedor provou a otimalidade para esta instância. Destacamos em negrito na primeira coluna o modelo que apresentou melhor resultado para cada classe em termos de tempo computacional médio (no caso da classe $3 \mathrm{~S}$, em que os tempos computacionais foram iguais, utilizou-se o GAP médio). 


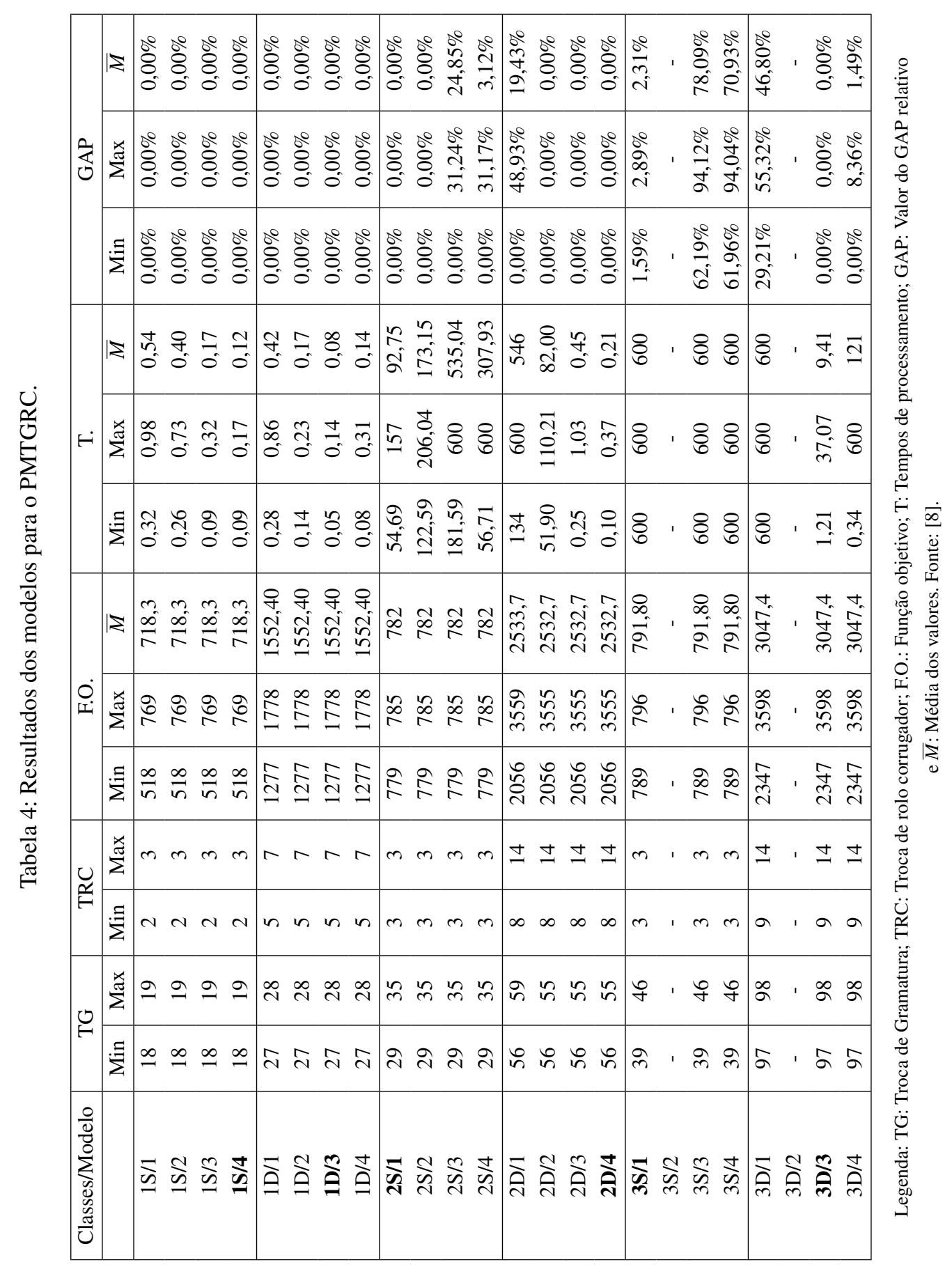


Em relação a classe 1S, a otimalidade foi provada para todas as instâncias da classe para os quatro modelos testados, sendo que os tempos de processamento foram inferiores a 1 segundo. Os menores tempos de processamento foram observados no modelo 4 e os maiores tempos no modelo 1, em média, o tempo de processamento do modelo 4 foi $77,7 \%$ menor, se comparado ao modelo 1. Para essa classe o modelo 4 apresentou os melhores resultados.

Em relação a classe 1D, em todas as instâncias e para todos os modelos foi provada a otimalidade, sendo os menores tempos de processamento observados no modelo 3 e os maiores tempos no modelo 1, sendo que os tempos foram inferiores a 1 segundo. Para essa classe o modelo 3 apresentou os melhores resultados.

Em relação a classe $2 \mathrm{~S}$, provou-se a otimalidade para todas as instâncias da classe para os modelos 1 e 2, sendo que o tempo de processamento para o modelo 1 foi em média $47 \%$ menor, se comparado ao modelo 2 . Utilizando o modelo 3 , foi provada a otimalidade para apenas duas instâncias, os tempos de processamento foram bem maiores se comparados aos modelos 1 e 2 e o GAP médio foi de $24,85 \%$. Já para o modelo 4, para 9 instâncias tem-se a prova de otimalidade, incluindo 7 instâncias para as quais não se tinha tal prova utilizando o modelo 3 , sendo que o GAP médio igual a $3,12 \%$. Em relação ao tempo, o modelo 1 consumiu 30,12\% do tempo consumido pelo modelo 4, em média. Para essa classe o modelo 1 apresentou os melhores resultados.

Em relação a classe 2D, para os modelos 2, 3 e 4 provou-se a otimalidade para todas as instâncias da classe, já para o modelo 1, para apenas duas instâncias. Em relação aos tempos de processamento, o modelo 4 apresentou os menores valores, sendo o valor médio igual a 0,21 segundos, bem inferior aos 546 segundos do valor médio obtido para o modelo 1. Os valores para o GAP, relativos ao modelo 1 , foram altos, atingindo um pico de $48,93 \%$ e uma média de $19,43 \%$. Para essa classe o modelo 4 apresentou os melhores resultados.

Em relação a classe 3S, não provou-se a otimalidade para nenhuma das instâncias, em nenhum dos modelos testados, o que deixou todos os tempos de processamento no limite de 600 segundos. Em relação ao GAP médio, o melhor resultado foi observado no modelo 1, com 2,31\%, seguido pelo modelo 4 com um valor de $70,93 \%$ e por fim temos o modelo 3 com valor médio de $78,09 \%$. Já para o modelo 2, devido as restrições de eliminação de sub-rotas DFJ, que apresentam crescimento exponencial, a memória do computador não foi suficiente para o processamento e não obteve-se nenhuma solução, o que indicamos por (-). Para essa classe o modelo 1 apresentou os melhores resultados.

Em relação a classe 3D, para o modelo 1, a otimalidade não foi provada para nenhuma das instâncias da classe, o GAP atingiu um pico de 55,32\% e uma média de 46,80\%. Para o modelo 2, que utiliza as restrições de eliminação de sub-rotas DFJ, ocorreu o mesmo que na classe $3 \mathrm{~S}$ anterior e não obtivemos nenhuma solução. Para o modelo 3 os resultados foram bem melhores, provou-se a otimalidade para todas as instâncias e tempo de processamento médio de 9,41 segundos. Para o modelo 4, para 8 instâncias foi provada a otimalidade, sendo que as duas instâncias sem otimalidade apresentaram GAPs iguais a $6,61 \%$ e $8,36 \%$. De um modo geral os tempos de processamento para o modelo 4 , foram mais baixos que os do modelo 3, mas o valor médio 
para o modelo 4 é bem maior devido as duas instâncias sem otimalidade que consumiram 600 segundos. Para essa classe o modelo 3 apresentou os melhores resultados.

Como os tempos de processamento para todos os modelos na classe $1 \mathrm{~S}$ são inferiores a 1 segundo e os modelos 3 e 4 tem praticamente o mesmo tempo de processamento na classe $2 \mathrm{D}$, se tivéssemos que eleger um modelo para cada tipo de papelão, poderíamos estabelecer o seguinte, utilizando as conclusões anteriores: o modelo 1 se mostrou eficiente para as instâncias cujos boletins tem chapas de parede simples, a saber, $1 \mathrm{~S}, 2 \mathrm{~S}$ e $3 \mathrm{~S}$ e o modelo 3 se mostrou eficiente para os instâncias cujos boletins tem chapas de parede dupla, a saber, 1D, 2D e 3D.

Como a prova da otimalidade está intimamente ligada à qualidade dos limitantes inferiores, analisamos também os valores das funções objetivo (F.O.) obtidos ao resolver as relaxações lineares para cada instãncia com cada modelo. Para tanto, resolvemos os modelos com todas as variáveis relaxadas para contínuas, ou seja, às variáveis binárias foram consideradas pertencentes ao intervalo $[0,1]$ e as variáveis inteiras (e positivas) foram consideradas como pertencentes ao reais (e positivas). Os resultados são mostrados na Tabela 5. A Tabela mostra que as relaxações lineares obtidas com o modelo 1 são iguais a zero para todas as instâncias. Em relação aos modelo 2 e 3 observa-se resultados semelhantes para as classes $1 \mathrm{D}$ e $2 \mathrm{D}$ e resultados melhores para o modelo 2 nas classes $1 \mathrm{~S}$ e $2 \mathrm{~S}$. Mesmo na relaxação linear, para o modelo 2 não obtemos resultados para as classes $3 \mathrm{~S}$ e 3D, devido ao crescimento exponencial das restrições de eliminação de sub-rotas DFJ. Em relação ao modelo 4, observa-se os melhores resultados para as 1S, 3S, 1D, 2D e 3D, dentre essas, destacamos as classes de papelão de parede dupla onde se obteve os melhores resultados, com destaque para a classe 1D, onde em 30\% das instâncias os valores das relaxações são iguais as soluções ótimas e em outros $30 \%$ diferem da solução ótima por apenas uma unidade. Observa-se que as soluções ótimas foram obtidas deixando os modelos rodarem no resolvedor CPLEX, sem limite de tempo. 


\begin{tabular}{|c|c|c|c|c|c|}
\hline 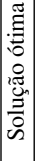 & 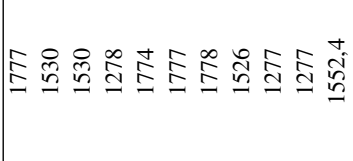 & 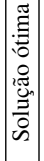 & 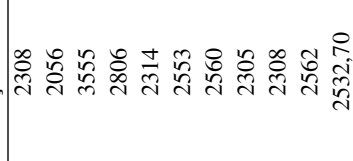 & 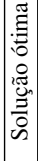 & 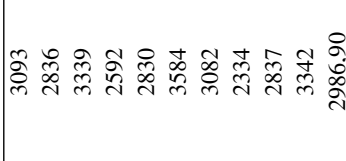 \\
\hline$\left|\begin{array}{c}+ \\
0 \\
\frac{0}{\pi} \\
\frac{0}{2} \\
\frac{2}{2}\end{array}\right|$ & 总 & 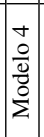 & 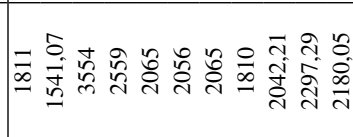 & $\mid \begin{array}{l}+ \\
\frac{0}{0} \\
\frac{\pi}{0} \\
\sum^{2}\end{array}$ & 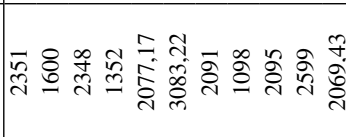 \\
\hline$\left|\begin{array}{c}0 \\
0 \\
\frac{0}{2} \\
\frac{0}{2} \\
\frac{0}{2}\end{array}\right|$ & 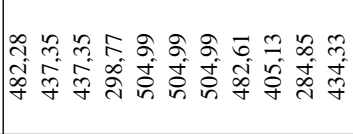 & $\mid \begin{array}{l}3 \\
\frac{0}{20} \\
\frac{\pi}{0} \\
\frac{0}{2} \\
\end{array}$ & 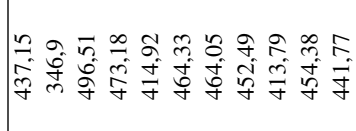 & $\begin{array}{l}m \\
0 \\
\frac{0}{2} \\
\frac{0}{2} \\
\sum\end{array}$ & 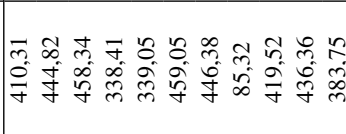 \\
\hline \begin{tabular}{|l|} 
\\
\\
0 \\
$\frac{\pi}{2}$ \\
\\
$\Sigma$ \\
\end{tabular} & 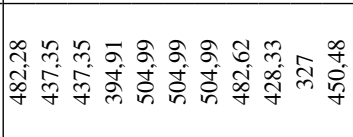 & 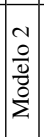 & 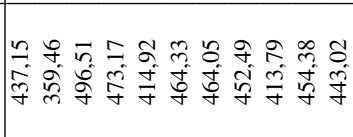 & $\mid \begin{array}{l}2 \\
\frac{0}{2} \\
\frac{0}{2} \\
\frac{D}{2}\end{array}$ & $* * * * * * * * * * *$ \\
\hline $\mid$\begin{tabular}{|c|} 
\\
0 \\
$\frac{0}{2}$ \\
\\
\\
\end{tabular} & 0000 & 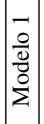 & 00000000000 & $\begin{array}{l}- \\
\frac{0}{2} \\
\frac{\bar{d}}{2} \\
\frac{0}{2}\end{array}$ & 00000000000 \\
\hline 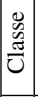 & 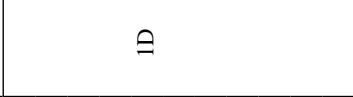 & 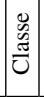 & तิ & $\begin{array}{l}\mathscr{\Xi} \\
\mathscr{E} \\
\tilde{\Xi}\end{array}$ & लि \\
\hline 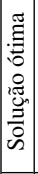 & 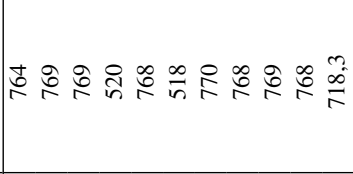 & 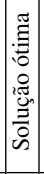 & 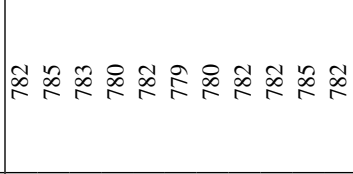 & 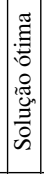 & 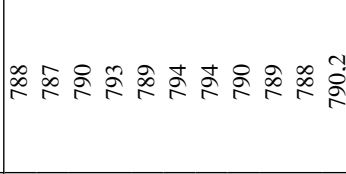 \\
\hline$\left|\begin{array}{l}+ \\
0 \\
\frac{0}{0} \\
\\
\\
\end{array}\right|$ & 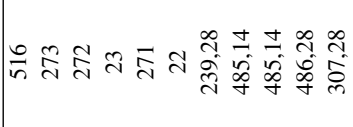 & 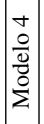 & ๓ & $\begin{array}{l}+ \\
0 \\
\frac{0}{0} \\
\frac{0}{0} \\
\Sigma \\
\Sigma\end{array}$ & 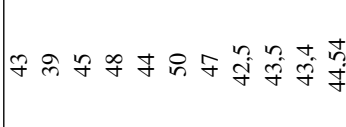 \\
\hline $\mid \begin{array}{l}m \\
0 \\
0 \\
\frac{0}{2} \\
\frac{0}{2} \\
\end{array}$ & 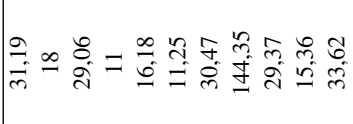 & $\begin{array}{l}m \\
\frac{0}{2} \\
\frac{0}{2} \\
\sum \\
\Sigma\end{array}$ & 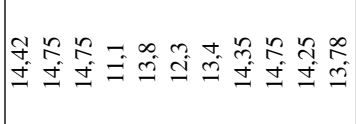 & $\begin{array}{c}m \\
0 \\
\frac{0}{2} \\
\frac{0}{0} \\
\sum \\
\Sigma\end{array}$ & 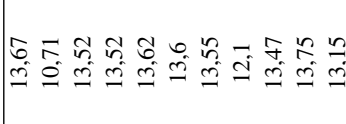 \\
\hline 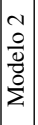 & 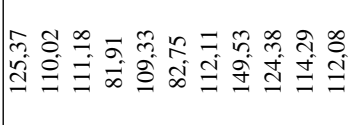 & 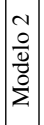 & 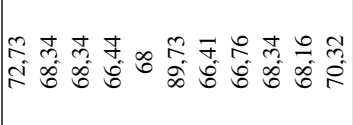 & $\left|\begin{array}{c}1 \\
0 \\
\frac{0}{\pi} \\
\frac{\pi}{2} \\
\end{array}\right|$ & $* * * * * * * * * * *$ \\
\hline 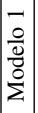 & 00000000000 & $\left|\begin{array}{l}-\frac{0}{2} \\
\frac{0}{2} \\
\frac{0}{2} \\
\sum_{2}\end{array}\right|$ & 00000000000 & $\left|\begin{array}{l}0 \\
0 \\
\frac{\pi}{0} \\
\overline{0} \\
\overline{2}\end{array}\right|$ & 00000000000 \\
\hline$\left|\begin{array}{l}\mathscr{y} \\
\tilde{z} \\
\tilde{U} \\
\end{array}\right|$ & 丞 & 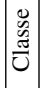 & $\frac{\sqrt[m]{7}}{2}$ & 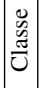 & : \\
\hline
\end{tabular}




\subsection{Comparando soluções da prática com as do modelo matemático}

Nesta seção, comparamos os valores obtidos na prática associados à troca de gramaturas, rolos corrugadores e valores de função objetivo para 20 conjuntos de boletins cujas as chapas foram processadas na IEPO visitada, com as soluções obtidas utilizando o Modelo 1. Esse modelo foi escolhido, pois obteve-se com ele os melhores resultados analisando boletins de parede simples, e os 20 conjuntos de boletins analisados na Tabela 6 são desse tipo. Além disso, em cada boletim são utilizados no máximo 2 tipos de onda e destacamos em negrito as classes onde o valor obtido pelo modelo foi melhor do que o utilizado na prática.

Tabela 6: Comparando soluções da prática com as do modelo matemático.

\begin{tabular}{|c|c|c|c|c|c|c|c|}
\hline Conjunto & Boletins & \multicolumn{2}{|c|}{ TG } & \multicolumn{2}{c|}{ TRC } & \multicolumn{2}{c|}{ F.O. } \\
\hline & & Prática & Modelo & Prática & Modelo & Prática & Modelo \\
\hline 1 & 5 & 9 & $\mathbf{7}$ & 1 & 1 & 259 & $\mathbf{2 5 7}$ \\
2 & 6 & 7 & $\mathbf{6}$ & 1 & 1 & 257 & $\mathbf{2 5 6}$ \\
3 & 7 & 13 & $\mathbf{1 0}$ & 1 & 1 & 263 & $\mathbf{2 6 0}$ \\
4 & 8 & 7 & 7 & 1 & 1 & 257 & 257 \\
5 & 8 & 13 & $\mathbf{1 0}$ & 1 & 1 & 263 & $\mathbf{2 6 0}$ \\
6 & 8 & 10 & $\mathbf{7}$ & 2 & $\mathbf{1}$ & 510 & $\mathbf{2 5 7}$ \\
7 & 8 & 14 & $\mathbf{1 1}$ & 2 & $\mathbf{1}$ & 514 & $\mathbf{2 6 1}$ \\
8 & 9 & 10 & $\mathbf{6}$ & 0 & 0 & 10 & $\mathbf{6}$ \\
9 & 9 & 18 & $\mathbf{1 3}$ & 0 & 0 & 18 & $\mathbf{1 3}$ \\
10 & 9 & 13 & $\mathbf{9}$ & 1 & 1 & 263 & $\mathbf{2 5 9}$ \\
11 & 10 & 21 & $\mathbf{1 6}$ & 2 & $\mathbf{1}$ & 521 & $\mathbf{2 6 6}$ \\
12 & 10 & 12 & $\mathbf{1 1}$ & 1 & 1 & 262 & $\mathbf{2 6 1}$ \\
13 & 10 & 13 & $\mathbf{9}$ & 1 & 1 & 263 & $\mathbf{2 5 9}$ \\
14 & 11 & 8 & $\mathbf{6}$ & 0 & 0 & 8 & $\mathbf{6}$ \\
15 & 11 & 21 & $\mathbf{1 5}$ & 1 & 1 & 271 & $\mathbf{2 6 5}$ \\
16 & 11 & 22 & $\mathbf{1 4}$ & 2 & $\mathbf{1}$ & 522 & $\mathbf{2 6 4}$ \\
17 & 12 & 8 & $\mathbf{5}$ & 0 & 0 & 8 & $\mathbf{5}$ \\
18 & 12 & 15 & $\mathbf{1 0}$ & 0 & 0 & 15 & $\mathbf{1 0}$ \\
19 & 12 & 18 & $\mathbf{1 2}$ & 2 & $\mathbf{1}$ & 518 & $\mathbf{2 6 2}$ \\
20 & 13 & 22 & $\mathbf{1 4}$ & 1 & 1 & 272 & $\mathbf{2 6 4}$ \\
\hline
\end{tabular}

Legenda: TG: Troca de gramatura; TRC: Troca de rolo corrugador; F.O: Função objetivo. Fonte: [8].

Os resultados para a F.O. atestam que as soluções obtidas via modelo são melhores que as utilizadas na indústria, sendo que para alguns conjuntos analisados, como o conjunto 16 e o 20 , houve redução de 37\% no número de trocas de gramaturas.

Em relação a troca de rolos corrugadores, quando a solução obtida é 0, indica que todos os boletins tem mesmo tipo de onda. Como temos no máximo dois tipos de onda e a relação entre 
os pesos das trocas de gramaturas e trocas de rolos corrugadores é 1:250, há um agrupamento dos boletins com mesmo tipo de onda, o que se observa na prática para a maioria dos conjuntos processados, por isso as soluções ótimas são iguais a 1. Todas as soluções obtidas via modelo são ótimas e os tempos de processamentos são inferiores a 2 segundos.

Para maior clareza, na Tabela 7 destacamos as gramaturas e o tipo de rolo corrugador empregado no conjunto 16 da Tabela 6, com o sequenciamento que foi utilizado na prática, sendo que os porta-bobinas foram indicados por PB e o rolo corrugador por RC.

Tabela 7: Sequenciamento dos boletins do conjunto 16 realizado na prática.

\begin{tabular}{|c|c|c|c|c|}
\hline Boletins & Gramatura PB 1 & Gramatura PB 2 & Gramatura PB 3 & Tipo de RC \\
\hline 1 & 120 & 120 & 140 & B \\
2 & 160 & 160 & 160 & B \\
3 & 160 & 160 & 160 & B \\
4 & 140 & 140 & 140 & B \\
5 & 120 & 120 & 120 & B \\
6 & 100 & 100 & 100 & B \\
7 & 160 & 180 & 160 & C \\
8 & 160 & 140 & 160 & B \\
9 & 140 & 120 & 120 & B \\
10 & 140 & 120 & 120 & B \\
11 & 200 & 200 & 200 & B \\
\hline Total de trocas & 7 & 8 & 7 & 2 \\
\hline
\end{tabular}

Legenda: PB: Porta-bobinas; RC: Rolo corrugador. Fonte: [8].

Somando-se o total de trocas em cada porta-bobinas, no sequenciamento da Tabela 7 obtemos 22 trocas de gramaturas e 2 trocas de rolo corrugador, o que implica num valor de 522 na função objetivo, que convertido em custos reais representa $22 \cdot \mathrm{R} \$ 9,46=\mathrm{R} \$ 208,12$ relativo a troca da gramaturas e $2 \cdot \mathrm{R} \$ 2.402,10=\mathrm{R} \$ 4.804,20$ relativo a troca de rolo corrugador, perfazendo um custo total de $\mathrm{R} \$ 5.012,32$.

Já na Tabela 8 trazemos o sequenciamento obtido pelo modelo 1, que gerou 14 trocas de gramaturas e 1 troca de rolo corrugador, o que implica num valor de 264 na função objetivo, que equivale a um custo total de $\mathrm{R} \$ 2.534,54$, ou seja, numa redução de 49,57\%, ou $\mathrm{R} \$ 2.477,78$.

Destacamos também na Tabela 8 que para o porta-bobinas 1, temos 5 tipos de gramaturas, o que fornece uma solução ótima igual a 4 para o número de trocas de gramaturas, já para o portabobinas 2 e para o porta-bobinas 3 temos respectivamente, 6 e 5 tipos de gramaturas, o que fornece um valor ótimo para o número de trocas de gramaturas, igual a 5 e 4 , valores esses que foram obtidos via modelo matemático. 
Tabela 8: Sequenciamento dos boletins do conjunto 16 utilizando o Modelo 1.

\begin{tabular}{|c|c|c|c|c|}
\hline & Gramatura PB 1 & Gramatura PB 2 & Gramatura PB 3 & Tipo de RC \\
\hline Boletim 11 & 200 & 200 & 200 & B \\
Boletim 6 & 100 & 100 & 100 & B \\
Boletim 10 & 140 & 120 & 120 & B \\
Boletim 9 & 140 & 120 & 120 & B \\
Boletim 5 & 120 & 120 & 120 & B \\
Boletim 1 & 120 & 120 & 140 & B \\
Boletim 4 & 140 & 140 & 140 & B \\
Boletim 8 & 160 & 140 & 160 & B \\
Boletim 2 & 160 & 160 & 160 & B \\
Boletim 3 & 160 & 160 & 160 & B \\
Boletim 7 & 160 & 180 & 160 & C \\
\hline Total de trocas & 5 & 5 & 4 & 1 \\
\hline
\end{tabular}

Legenda: PB: Porta-bobinas; RC: Rolo corrugador. Fonte: [8].

\section{CONCLUSÕES E TRABALHOS FUTUROS}

Neste trabalho estendemos alguns modelos da literatura, propostos para o Problema de Minimização de Trocas de Ferramentas, para o Problema de Minimização de Trocas de Gramaturas e Rolos Corrugadores que surge em indústrias de embalagens de papelão ondulado. Até onde sabemos, a extensão proposta é inédita e, adapta um problema clássico da literatura para o caso em que as ferramentas tem que ser posicionadas em determinado local específico do magazine que, no problema tratado, equivale ao porta-bobinas relacionado à camada que será produzida. Os modelos propostos são comparados entre si e com a prática. Os resultados computacionais indicam que o modelo 1 se mostrou mais eficiente para as instâncias cujos boletins tem chapas de parede simples, e o modelo 3 se mostrou eficiente para os instâncias cujos boletins tem chapas de parede dupla. Além disso, na comparação com a prática os resultados obtidos pelo modelo 1 indicam que é possível reduzir em até $37 \%$ o número de trocas de gramaturas, mostrando a superioridade do modelo. Portanto, os resultados computacionais mostram que os modelos tem potencial para serem utilizados na prática.

Acreditamos que existem várias possibilidades para serem exploradas futuramente, tanto do ponto de vista teórico em que se tem uma extensão de um problema clássico, como do ponto de vista prático encontrado em indústrias de embalagens de papelão. Mais especificamente, como trabalhos futuros, pretende-se analisar os avanços propostos na literatura para o Problema de Minimização de Trocas de Ferramentas ([24], [20], entre outros) e adapta-los, quando possível, para o problema tratado neste trabalho. Em relação à aplicação às indústrias de embalagens de papelão ondulado, pretende-se considerar uma extensão do problema em que, no caso de boletins de 3 camadas, diferentes combinações dos 5 porta-bobinas possam ser utilizadas e não somente 
os 3 primeiros. Por fim, a consideração de outros objetivos da indústria de maneira acoplada a este problema pode ser considerada futuramente.

\title{
Agradecimentos
}

S. A. de Araujo agradece ao CNPq e à FAPESP (Processos 2016/01860-1 e 2013/07375-0) pelo suporte financeiro.

\begin{abstract}
In this paper we address a real problem identified in the production process of corrugated packaging industries. The Production Planning and Control sector issues bulletins that bring the characteristics related to the sheets that will be produced. The production of these sheets is done in the corrugator, where reels are installed, according to the characteristics of the bulletins. The problem arises from the need to minimize the switches of weights and corrugator rolls during the sheets production process, since each bulletin brings the specific weight of the layers (usually 3 or 5 ) of the sheet, in addition to the type of wave used. The sequencing of bulletins impacts the amount of weight and corrugator rolls switches. We interpreted this problem as a Minimization of Tool Switches Problem and we present four mathematical models for the case under study. Some computational results are presented comparing the results obtained by the proposed mathematical models and by a factory of the sector, and that it is possible to reduce up to $37 \%$ the number of switches of weights.
\end{abstract}

Keywords: mixed-integer programming, minimization of tool switches, corrugated packaging industries.

\section{REFERÊNCIAS}

[1] J.C. Becceneri, H.H. Yanasse \& N.Y. Soma. A method for solving the minimization of the maximum number of open stacks problem within a cutting process. Computers \& Operations Research, 31(14) (2004), 2315-2332.

[2] A. Bolat. An extended scheduling model for producing corrugated boxes. International Journal of Production Research, 38(7) (2000), 1579-1599.

[3] D. Catanzaro, L. Gouveia \& M. Labbé. Improved integer linear programming formulations for the job sequencing and tool switching problem. European journal of operational research, 244(3) (2015), 766-777.

[4] C. Chantrapornchai \& T. Sathapanawat. Corrugation Software Development and Deployment: A Case Study. International Journal of u-and e-Service, Science and Technology, 5(1) (2012), 1-24.

[5] Y. Crama, A.G. Oerlemans \& F.C. Spieksma. Minimizing the number of tool switches on a flexible machine. In "Production Planning in Automated Manufacturing". Springer (1994), pp. 165-195.

[6] G. Dantzig, R. Fulkerson \& S. Johnson. Solution of a large-scale traveling-salesman problem. Journal of the operations research society of America, 2(4) (1954), 393-410. 
[7] M. Desrochers \& G. Laporte. Improvements and extensions to the Miller-Tucker-Zemlin subtour elimination constraints. Operations Research Letters, 10(1) (1991), 27-36.

[8] M. Ferreira. "Problemas de programação de produção em indústrias de embalagens de papelão ondulado.”. Ph.D. thesis, Universidade Estadual Paulista (UNESP) (2018).

[9] M. Ferreira, V.C.B. Camargo \& S.A. de Araujo. The reel allocation problem of a corrugated packaging company. Pesquisa Operacional, 40(e229708) (2020), 1-21.

[10] R. Fourer, D.M. Gay \& B.W. Kernighan. "AMPL: a Modeling Language for Mathematical Programming". Duxbury Press (2002).

[11] R.W. Haessler \& F.B. Talbot. A 0-1 model for solving the corrugator trim problem. Management science, 29(2) (1983), 200-209.

[12] A. Hinxman. The trim-loss and assortment problems: A survey. European Journal of Operational Research, 5(1) (1980), 8-18.

[13] IBM ILOG. "IBM ILOG CPLEX Optimization Studio CPLEX User's Manual”. IBM Corp. 1987, 2017 (2017).

[14] G. Laporte, J.J. Salazar-Gonzalez \& F. Semet. Exact algorithms for the job sequencing and tool switching problem. IIE Transactions, 36(1) (2004), 37-45.

[15] S. Lins. CONJUG: Um Sistema para diminuir perdas no corte industrial de chapas, barras, bobinas. The Proceedings of the XXI Congresso Nacional de Informatica., (1988).

[16] S. Lins. Traversing trees and scheduling tasks for duplex corrugator machines. Pesquisa Operacional, 9(1) (1989), 40-54.

[17] K. Matsumoto, H. Miwa \& T. Ibaraki. Scheduling of corrugated paper production. European Journal of Operational Research, 192(3) (2009), 782-792.

[18] C.E. Miller, A.W. Tucker \& R.A. Zemlin. Integer programming formulation of traveling salesman problems. Journal of the ACM (JACM), 7(4) (1960), 326-329.

[19] R. Morábito. Modelos de otimização para o problema de corte nas indústrias de papel e papelão e de móveis. Gestão \& Produção, 1(1) (1994), 59-76.

[20] A.C.B. Moreira. "O problema de minimização de trocas de ferramentas". Ph.D. thesis, Universidade de São Paulo (2016).

[21] M.A. Rodríguez \& A. Vecchietti. An efficient model implementation to solve a real-world cutting stock problem for a corrugated board boxes mill. Computer Aided Chemical Engineering, 24 (2007), 601.

[22] M. Savsar \& C. Cogun. Analysis and modelling of a production line in a corrugated box factory. The International Journal of Production Research, 32(7) (1994), 1571-1589.

[23] C.S. Tang \& E.V. Denardo. Models arising from a flexible manufacturing machine, part 1: minimization of the number of tool switches. Operations research, 36(5) (1988), 767-777. 
[24] H.H. Yanasse, R.d.C.M. Rodrigues \& E.L.F. Senne. Um algoritmo enumerativo baseado em ordenamento parcial para resolução do problema de minimização de trocas de ferramentas. Gestão \& Produção, (2009), 370-381.

(cc) BY 